\title{
CRITERIA FOR COMPACTNESS AND FOR DISCRETENESS OF LOCALLY COMPACT AMENABLE GROUPS
}

\author{
EDMOND GRANIRER ${ }^{1}$
}

ABSTRACT. Let $G$ be a locally compact group $P(G)=\{0 \leqq \phi \in$ $\left.L_{1}(G) ; \int \phi(x) d x=1\right\}$ and $\left(l_{a} f\right)(x)={ }_{a} f(x)=f(a x)$ for all $a, x \in G$ and $f \in L^{\infty}(G) .0 \leqq \Psi^{*} \in L^{\infty}(G)^{*}, \Psi^{*}(1)=1$ is said to be a [topological] left invariant mean ([TLIM] LIM) if $\left.\Psi_{(a} f\right)=\Psi^{*}(f)\left[\Psi^{*}(\phi * f)=\right.$ $\Psi(f)]$ for all $a \in G, \phi \in P(G), f \in L^{\infty}(G)$. The main result of this paper is the

THEOREM. Let $G$ be a locally compact group, amenable as a discrete group. If $G$ contains an open $\sigma$-compact normal subgroup, then $L I M=T L I M$ if and only if $G$ is discrete. In particular if $G$ is an infinite compact amenable as discrete group then there exists some $\Psi \in L I M$ which is different from normalized Haar measure. A harmonic analysis type interpretation of this and related results are given at the end of this paper. ${ }^{2}$

Introduction. It was known to Fred Greenleaf that if $T$ is the circle group then there are at least two different linear translation invariant functionals $\Psi \geqq 0$ on $L^{\infty}(T)$ with $\Psi(1)=1$. One of them is certainly that given by the normalized Haar measure $\lambda$ on $T$.

It is easy to show and it is known that on any compact $G, \lambda$ is the unique $0 \leqq \Psi \in L^{\infty}(G)^{*}, \Psi 1=1$ which satisfies the stronger invariance property $\Psi^{(}(\phi * f)=\Psi^{\prime}(f)$ for all $f \in L^{\infty}(G), \phi \in P(G)$ (i.e. $\lambda$ is the unique TLIM on $\left.L^{\infty}(G)\right)$. This is the case since $\phi * f \in C(G)$ for all $\phi \in P(G)$, $f \in L^{\infty}(G)$ and if $\Psi \in$ TLIM then $\Psi \in \operatorname{LIM}[6$, p. 25]. Thus $\Psi=\lambda$ at least on $C(G)$. But then for all $f \in L^{\infty}(G), \Psi(f)=\Psi(\phi * f)=\lambda(\phi * f)=\lambda(f)$.

It seemed to Greenleaf that for any compact infinite $G$, which is amenable as a discrete group, there exist at least two different LIM's on $L^{\infty}(G)$. Our main result in this paper implies the

THEOREM. Let $G$ be a locally compact group which is abelian or $\sigma$-compact and amenable as a discrete group. Then $L I M=T L I M$ if and

Received by the editors September 18, 1972.

AMS (MOS) subject classifications (1970). Primary 22D15, 22D35, 43A07; Secondary 22D05, 43A20.

1 This research was done while the author held a Canada Council award. It is gratefully acknowledged.

Thanks are due to the referee for a simplification in the proof of Proposition 1.

${ }^{2}$ The main result of this paper has independently been obtained by $W$. Rudin in a recent paper Invariant means on $L^{\infty}$, Studia Math. 44 (1972), 219-227, which was not in print when our paper was sent for publication. (See Addition at the end of present paper.) 
only if $G$ is discrete. In particular on any compact infinite $G$ which is amenable as discrete there exists some $\Psi \in L I M$ different from the normalized Haar measure.

Let $H\left[H_{c}\right]$ be the linear span of $\left\{f-l_{a} f ; f \in L^{\infty}(G), a \in G\right\}[\{f-\phi * f$; $\left.\left.\phi \in P(G), f \in L^{\infty}(G)\right\}\right]$ and for $A \subset L^{\infty}(G)$ let $\bar{A}\left[\bar{A}^{*}\right]$ denote the norm [w*] closure of $A$ in $L^{\infty}(G)$. In any locally compact group one has $\bar{H} \subset \bar{H}_{c} \subset \bar{H}_{c}^{*}=\bar{H}^{*} \subset L^{\infty}(G)$. Our last result (combined with some known facts) when restricted to $\sigma$-compact locally compact abelian groups runs as follows:

Proposition. (i) If $G$ is compact and infinite then $\bar{H} \subsetneq \bar{H}_{c}=\bar{H}_{c}^{*}=\bar{H}^{*}=$ $\left\{f \in L^{\infty}(G) ; \lambda f=0\right\}$.

(ii) If $G$ is not compact then $\bar{H} \subset \bar{H}_{c} \subset \bar{H}_{c}^{*}=\bar{H}^{*}=L^{\infty}(G)$. Moreover $L^{\infty}(G) / \bar{H}_{c}$ is a nonseparable Banach space and $\bar{H}=\bar{H}_{c}$ iff $G$ is discrete.

We conjecture at the end that for any locally compact amenable group $G$, if $G$ is noncompact then $L^{\infty}(G) / \bar{H}_{c}$ is a nonseparable Banach space and if $G$ is nondiscrete then $\bar{H}_{c} / \bar{H}$ is nonseparable (with induced quotient norms).

Some more notations. Unless otherwise specified we assume the notations and definitions of Hewitt-Ross [7].

If $G$ is a locally compact group $\lambda$ will denote a fixed left Haar measure (with $\lambda(G)=1$ if $G$ is compact), we write sometimes $\int \phi(x) d x$ instead of $\int \phi d \lambda$.

$\Psi \in L^{\infty}(G)^{*}$ is said to be [topologically] left invariant if $\Psi\left(l_{a} f\right)=$ $\Psi(f) \quad[\Psi(\phi * f)=\Psi(f)]$ for all $f \in L^{\infty}(G), \quad \phi \in P(G), \quad a \in G$ (where $l_{a} f(x)={ }_{a} f(x)=f(a x)$ ). If $\Psi$ satisfies in addition $\Psi \geqq 0$ and $\Psi(1)=1$ then $\Psi^{\circ}$ is said to be a [topological] left invariant mean ([TLIM] LIM resp.). The set of all [TLIM] LIM is also denoted by [TLIM] LIM. Analogously we define [TRIM] RIM the sets of [topological] right invariant means.

We stress that LIM, TLIM are both included in $L^{\infty}(G)^{*}$. The locally compact group $G$ is said to be amenable if LIM $\neq \varnothing$ (or equivalently if TLIM $\neq \varnothing$ see [6]). $G$ is said to be amenable as discrete if $G_{d}$ (i.e. $G$ with the discrete topology) is amenable.

We write sometimes $\operatorname{LIM}(G), \operatorname{TLIM}(G)$ to emphasize dependence on the group $G$. If $A \subset G, 1_{A}$ denotes the function 1 on $A$ and zero otherwise. If $\Psi \in L^{\infty}(G)^{*}$, we write $\Psi(B)$ instead of $\Psi^{*}\left(1_{B}\right)$ for measurable $B \subset G$. 1 also stands for the constant one function on $G$.

Proposition 1. Let $G$ be any noncompact locally compact group and $\phi \in T R I M$. If $B$ is a measurable set and $\lambda(B)<\infty$ then $\phi(B)=0$. 
Proof. Let $\phi_{\alpha} \in P(G)$ be such that $\phi_{\alpha} \rightarrow \phi$ in $w^{*}$ and let $\eta \in P(G)$ be such that $0 \leqq \eta(x) \leqq \varepsilon$ for all $x \in G$. Then

$$
\left|\phi_{\alpha} * \eta(x)\right|=\left|\int \phi_{\alpha}(y) \eta\left(y^{-1} x\right) d \lambda\right| \leqq \varepsilon \int \phi_{\alpha}(y) d \lambda=\varepsilon .
$$

Furthermore if $f \in L^{\infty}(G)$ then

$$
\left(\phi_{\alpha} * \eta\right)(f)=\phi_{\alpha}(f * \tilde{\eta}) \rightarrow \phi(f * \tilde{\eta})=\phi(f) .
$$

(See Wong [10, p. 352].) Hence if $f \in L^{\infty} \cap L^{1}$ then $\left|\left(\phi_{\alpha} * \eta\right)(f)\right| \leqq \int \varepsilon|f| d \lambda$ so $|\phi f| \leqq\left(\int|f| d \lambda\right) \varepsilon$. Thus $\phi f=0$.

We need the following, probably known, proposition for which we were unable to find a reference.

Proposition 2. Let $G$ be a o-compact nondiscrete locally compact group. Then for any $\varepsilon>0$ there exists an open dense set $B \subset G$ with $\lambda(B)<\varepsilon$.

Proof. It is enough to show the existence of a dense set $D \subset G$ with $\lambda(D)=0$ and the regularity of $\lambda$ would imply that for some open $D \subset B$, $\lambda(B)<\varepsilon$.

If $G$ is separable then there is some countable dense $D \subset G$. Clearly $\lambda(D)=0$.

Assume now that $G$ is arbitrary and $N \subset G$ a closed normal subgroup. Let $\theta: G \rightarrow G / N$ be the canonical map. If $D \subset G$ with $\theta D$ dense in $G / N$ then $D N$ is dense in $G$. In fact if $U \subset G$ is open with $U \cap D N=\varnothing$ then $U N \cap D N$ $=\varnothing$ so $\theta^{-1}(\theta U \cap \theta D)=U N \cap D N=\varnothing$ thus $\theta U \cap \theta D=\varnothing$ and $\theta U$ is open in $G / N$ which cannot be.

If $G$ is $\sigma$-compact nondiscrete let $U \subset G$ be an open neighborhood of the identity and let $G_{0}=\bigcup_{-\infty}^{\infty} U^{n}$. Then $G_{0}$ is open compactly generated and there are countably many left cosets of $G$ w.r.t. $G_{0}$. The left Haar measure of $G_{0}$ can be taken to be the restriction to $G_{0}$ of the left Haar measure $\lambda$ on $G$. It is enough hence to show that there is a dense null set $D \subset G_{0}$ i.e. we can and shall assume that $G$ is compactly generated nondiscrete. Let then $U_{n}$ be a sequence of identity neighborhoods in $G$ with $\lambda\left(U_{n}\right) \rightarrow 0$ and let $N \subset \bigcap_{1}^{\infty} U_{n}$ be a compact normal subgroup such that $G / N$ is metrizable separable (see [7, p. 71]). $(G / N$ is not discrete since $\lambda N=0$ so $N$ is not open.) Let $D=\left\{d_{i}\right\}_{1}^{\infty} \subset G$ be such that its image in $G / N$ is dense. Then $D N \subset G$ is dense and $\lambda(D N)=0$ since $D$ is countable.

We need in the sequel the following proposition (not in its full force) which is in part due to Følner [3] for discrete amenable groups.

Proposition 3. Let $G$ be a locally compact group which is amenable as a discrete group. For $f \in L^{\infty}(G)$ let $M(f)=\sup \{\phi(f) ; \phi \in L I M\}$. Then 
for all $f \in L^{\infty}(G)$

$$
M f=\inf _{\mathscr{q}} \operatorname{ess} \sup _{x}\left[\frac{1}{n} \sum_{1}^{n} f\left(a_{i} x\right)\right]
$$

the inf being taken over the set $\mathscr{A}$ of all finite tuples $\left(a_{1}, \cdots, a_{n}\right)$ of elements of $G$.

Proof. Let $H$ be the linear span of $\left\{f-l_{a} f ; a \in G, f \in L^{\infty}(G)\right\}$. It is known (and due to Følner [3, p. 6] for discrete $G$ ) that:

$$
M(f)=\inf _{h \in H} \text { ess } \sup (f(x)+h(x))
$$

for all $f \in L^{\infty}(G)$. (For an easy proof see $[5$, p. 401].)

Also if $\phi \in$ LIM then $\phi f=\phi\left(n^{-1} \sum_{1}^{n} l_{a_{i}} f\right)$ hence

$$
M f \leqq \inf _{\mathscr{\alpha}} \operatorname{ess} \sup _{x} \frac{1}{n} \sum_{1}^{n} f\left(a_{i} x\right) .
$$

Let now $\varepsilon>0$ and $h_{0} \in H$ be such that $M(f)+\varepsilon>\operatorname{ess} \sup _{x}\left(f(x)+h_{0}(x)\right)$. So $M(f)+\varepsilon \geqq f(x)+h_{0}(x)$ locally a.e. and a fortiori $M(f)+\varepsilon \geqq$ $n^{-1} \sum_{1}^{n} l_{a_{i}}\left(f(x)+h_{0}(x)\right)$ loc. a.e. for all $a_{1}, \cdots, a_{n}$ in $G$. We claim that a finite set $\left\{b_{1}, \cdots, b_{k}\right\} \subset G$ can be chosen such that $\left|k^{-1} \sum l_{b_{i}} h_{0}(x)\right|<\varepsilon / 2$ loc. a.e. This would imply that $M(f)+3 / 2 \varepsilon \geqq k^{-1} \sum l_{b_{i}} f(x)$ loc. a.e., i.e. that

which would end this proof.

$$
M(f) \geqq \inf _{\mathscr{A}} \operatorname{ess} \sup _{x} \frac{1}{n} \sum_{1}^{n} l_{a_{i}} f(x)
$$

To prove this claim let $h_{0}=\sum_{1}^{n}\left[f_{i}-l_{c_{i}} f_{i}\right]$. For the finite set $F=$ $\left\{c_{1}, \cdots c_{n}\right\}$ choose a finite subset $A=\left\{b_{1}, \cdots, b_{k}\right\}$ to satisfy $c\left(c_{i} A \sim A\right)<$ $\delta c(A)$ for $1 \leqq i \leqq n$ where $c(B)$ stands for the cardinality of $B$ and $\delta=$ $\varepsilon\left(\max _{1 \leqq i \leqq n}\left\|f_{i}\right\|\right)^{-1} n^{-1}$. Such $A$ can be found by Følner's characterization of discrete amenable groups [2] (see Namioka [9, p. 22]). Then for each $i \leqq n$

$$
\begin{aligned}
\left|\frac{1}{k_{j}} \sum_{=1}^{k} l_{b_{j}}\left(f_{i}-l_{c_{i}} f_{i}\right)\right| & =\left|-\frac{1}{k} \sum_{j=1}^{k}\left(l_{c_{i} b_{j}}-l_{b_{j}}\right) f_{i}\right| \\
& \leqq c\left(c_{i} A-A\right)\left\|f_{i}\right\| / c(A)<\delta\left\|f_{i}\right\| \leqq \varepsilon / n .
\end{aligned}
$$

Therefore $\left|k^{-1} \sum l_{b_{i}} h_{0}(x)\right| \leqq \varepsilon / 2$ loc. a.e. which finishes this proof.

Remarks. 1. It seems that this proposition does not hold true if $G$ is not amenable as a discrete group (even in the case that $G$ is compact).

2. If $m(f)=\inf \{\phi(f) ; \phi \in \operatorname{LIM}\}$ then

$$
m(f)=-M(-f)=\sup _{\mathscr{A}}\left[\underset{x}{\operatorname{ess} \inf } \frac{1}{n} \sum_{1}^{n} f\left(a_{i} x\right)\right] .
$$


3. One can show in a similar way that the support functional of the

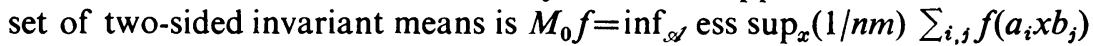
where $\mathscr{A}$ is the set of all pairs of finite tuples $\left(a_{1}, \cdots, a_{n}\right)\left(b_{1}, \cdots, b_{m}\right)$ of elements of $G$.

THEOREM 1. Let $G$ be a locally compact $\sigma$-compact group which is amenable as a discrete group. If LIM =TLIM then $G$ is discrete.

Proof. Assume that $G$ is not discrete and let $O$ be an open dense set in $G$ with $\lambda(O)<\frac{1}{2}$. Thus, if $G$ is not compact then $\phi(O)=0$ for all $\phi \in$ TRIM hence $\Psi\left(O^{-1}\right)=0$ for all $\Psi \in$ TLIM (see [4, p. 50]). If $G$ is compact then $\lambda\left(O^{-1}\right)=\lambda(O)<\frac{1}{2}$. Let $B=G \sim O^{-1}$. Then $B$ is closed nowhere dense, $\Psi(B)=1$ if $\Psi \in$ TLIM and $G$ is not compact while $\lambda(B)>\frac{1}{2}$ if $G$ is compact. (In this last case $\{\lambda\}=$ TLIM $=$ TRIM.) In different terminology $B$ is topologically left almost convergent to 1 (or to a positive real $>\frac{1}{2}$ if $G$ is compact).

We claim that $\phi(B)=0$ for some $\phi \in$ LIM. If not, then

$$
m\left(1_{B}\right)=\inf \left\{\phi\left(1_{B}\right) ; \phi \in \mathrm{LIM}\right\}=\sup _{\mathscr{\alpha}} \underset{x}{\operatorname{ess} \inf } \frac{1}{n} \sum_{1}^{n} 1_{B}\left(a_{i} x\right)=d>0
$$

But then, there are $b_{1}, \cdots, b_{k}$ in $G$ such that ess $\inf _{x} k^{-1} \sum_{1}^{k} 1_{B}\left(b_{j} x\right) \geqq d / 2$ i.e. locally a.e. in $x$ one has $k^{-1} \sum_{1}^{k} 1_{b_{j}-1}(x) \geqq d / 2>0$. But this contradicts the fact that $A=G \sim \bigcup_{1}^{k} b_{j}^{-1} B$ is open dense, hence of nonzero Haar measure and for $x \in A, k^{-1} \sum_{1}^{k} 1_{B}\left(b_{j} x\right)=0$. Using Remark 3 above one could easily show that in fact $\phi(B)=0$ for some two sided invariant mean $\phi$ on $L^{\infty}(G)$.

REMARKS. Let $G$ be a locally compact amenable group with $G_{0} \subset G$ an open subgroup. Let $\lambda\left(\lambda_{0}\right)$ be the Haar measures on $G\left(G_{0}\right)$. As known and easily shown the $\lambda_{0}$ measurable sets comprise exactly the $\lambda$ measurable sets of $G$ which are included in $G_{0}$. We can and shall choose $\lambda_{0}$ to be the restriction of $\lambda$ to $G_{0}$. (We use the terminology of [7].)

For $f \in L^{\infty}(G)$ define $(\pi f)(x)=f(x)$ for $x \in G_{0}$. Then $\pi$ can be considered as a map onto $L^{\infty}\left(G_{0}\right)$. If $v \in L^{\infty}\left(G_{0}\right) *$ is left invariant and $f \in L^{\infty}(G)$, let $\left(S_{v} f\right)(z)=v\left(\pi l_{z} f\right)$ for all $z \in G$. Let $\left\{z_{\alpha} G_{0}\right\}_{\alpha \in I}$ be a fixed decomposition of $G$ into left cosets w.r.t. $G_{0}$. Then, the bounded function, $S_{v} f$ is constant on each $z_{\alpha} G_{0}$ (as known) since if $z=z_{\alpha} a, a \in G_{0}$ then $S_{v} f\left(z_{\alpha} a\right)=v\left(\pi l_{z_{\alpha} a} f\right)=$ $v\left(l_{a}\left(\pi l_{z_{\alpha}} f\right)\right)=S_{v} f\left(z_{\alpha}\right)$, since $a \in G_{0}$. This implies that $S_{v} f \in U C B_{l}(G)$ (i.e. is left uniformly continuous as in [7] for all $f \in L^{\infty}(G)$ and left invariant $v \in L^{\infty}\left(G_{0}\right)^{*}$. This is the case since for all $z \in G, x \in G_{0}, S_{v} f(z x)-S_{v} f(z)=0$ and $G_{0}$ is open.

Choose and fix now some LIM, $\mu_{0}$ on $C(G)$ and define for any left invariant $v \in L^{\infty}\left(G_{0}\right)^{*}, T \nu \in L^{\infty}(G)^{*}$, by $T \nu(f)=\mu_{0}\left(S_{v} f\right)$. 
As known and readily checked $T$ maps the set of left invariant elements [LIM] of $L^{\infty}\left(G_{0}\right)^{*}$ into the set of left invariant elements [LIM] of $L^{\infty}(G)^{*}$. The above is a refinement of a construction due to M. M. Day [1, p. 533]. In the above context we have the

Proposition 4. Let $G$ be a locally compact amenable group and $G_{0} \subset G$ an open normal subgroup.

If $T v \in \operatorname{TLIM}(G)$ for some $v \in \operatorname{LIM}\left(G_{0}\right)$ then $v \in \operatorname{TLIM}\left(G_{0}\right)$.

Proof. If $f \in L^{\infty}\left(G_{0}\right)$ denote by $f_{1}$ its $\left\{z_{\alpha}\right\}$ periodic extension i.e. $f_{1}\left(z_{\alpha} x\right)=f(x)$ for all $x \in G_{0}$ and all $\alpha$. (Note that $\left\{z_{\alpha}\right\}$ are fixed.) It is clear that $f_{1}$ is measurable (since it needs to be so only on compacta [7, p. 131], and $G_{0}$ is open).

If $z \in z_{\alpha} G_{0}$ then

$$
S_{v}\left(f_{1}\right)(z)=S_{v}\left(f_{1}\right)\left(z_{\alpha}\right)=v\left(\pi l_{z_{\alpha}} f_{1}\right)=v(f)
$$

since if $x \in G_{0}$ then $\left(\pi l_{z_{\alpha}} f_{1}\right)(x)=f_{1}\left(z_{\alpha} x\right)=f(x)$. Thus $(T v) f_{1}=\mu_{0}\left(S_{v} f_{1}\right)=$ $\mu_{0}\left(v(f) \cdot 1_{G}\right)=v f$.

Fix now $\phi_{0} \in P(G)$ with support included in $G_{0}$. Then for $f \in L^{\infty}\left(G_{0}\right)$ and $x \in G_{0}$ one has:

$$
\begin{aligned}
l_{z_{\alpha}}\left(\phi_{0} * f_{1}\right)(x) & =\int f_{1}\left(y^{-1} z_{\alpha} x\right) \phi_{0}(y) d y \\
& =\int f_{1}\left(\left(z_{\alpha} y z_{\alpha}^{-1}\right)^{-1} z_{\alpha} x\right) \phi_{0}\left(z_{\alpha} y z_{\alpha}^{-1}\right) \Delta\left(z_{\alpha}^{-1}\right) d y \\
& =\int_{G_{0}} f_{1}\left(z_{\alpha} y^{-1} x\right) \phi_{0}\left(z_{\alpha} y z_{\alpha}^{-1}\right) \Delta\left(z_{\alpha}^{-1}\right) d y \\
& =\int_{G_{0}} f\left(y^{-1} x\right) \phi_{0}\left(z_{\alpha} y z_{\alpha}^{-1}\right) \Delta\left(z_{\alpha}^{-1}\right) d y=\left(\Psi_{\alpha} \circledast f\right)(x)
\end{aligned}
$$

where $\Psi_{\alpha}(y)=\phi_{0}\left(z_{\alpha} y z_{\alpha}^{-1}\right) \Delta\left(z_{\alpha}^{-1}\right)$ for $y \in G_{0}$, thus $\Psi_{\alpha} \in P\left(G_{0}\right)$ and where $\circledast$ stands for convolution in $L_{1}\left(G_{0}\right)$. Note, that since $G_{0}$ is normal $\phi_{0}\left(z_{\alpha} y z_{\alpha}^{-1}\right)$ has support included in $G_{0}$.

It follows that if $z \in z_{\alpha} G_{0}$ then

$$
\begin{aligned}
S_{v}\left(\phi_{0} * f_{1}\right)(z) & =S_{v}\left(\phi_{0} * f_{1}\right)\left(z_{\alpha}\right) \\
& =v \pi l_{z_{\alpha}}\left(\phi_{0} * f_{1}\right)=v\left(\Psi_{\alpha} \circledast f\right)=v\left(\phi_{0} \circledast f\right) .
\end{aligned}
$$

Note that we have used in the last equality only the fact that $v \in \operatorname{LIM}\left(G_{0}\right)$. From it alone, it follows (see Greenleaf [6, proof of Lemma 222, p. 27]) that $\nu(\phi \circledast f)=v(\Psi \circledast f)$ for all $\phi, \Psi \in P\left(G_{0}\right)$.

Hence $T v\left(\phi_{0} * f_{1}\right)=\mu_{0}\left(S_{v}\left(\phi_{0} * f_{1}\right)\right)=v\left(\phi_{0} \circledast f\right)$. 
But by assumption $T v \in$ TLIM. Thus $T \nu\left(\phi_{0} * f_{1}\right)=(T v) f_{1}=v f$ and hence, for all $f \in L^{\infty}\left(G_{0}\right), v\left(\phi_{0} \circledast f\right)=v(f)$. The above remark implies that $v \in \operatorname{TLIM}\left(G_{0}\right)$ and finishes this proof.

THEOREM 2. Let $G$ be a locally compact group which is amenable as a discrete group. Assume that $G$ contains a $\sigma$-compact open normal subgroup. If $\operatorname{LIM}(G)=T L I M(G)$ then $G$ is discrete.

RemarK. 1. If $G$ has equivalent left and right uniform structures then $G$ contains a neighborhood $U$ of the identity with compact closure such that $x U x^{-1}=U$ for all $x \in G$. Thus $G_{0}=\bigcup_{-\infty}^{\infty} U^{n}$ is normal $\sigma$-compact and open. In particular the theorem certainly holds true for all locally compact abelian groups $G$. It also holds true for all $\sigma$-compact $G$ which are amenable as discrete groups (take $G=G_{0}$ ).

2. We could have assumed in this theorem that $G$ is a locally compact amenable group and the open normal $\sigma$-compact $G_{0}$ is amenable as discrete. This however readily implies that $G$ is amenable as discrete and we would not gain anything. (The discrete $G / G_{0}$ and $G_{0}$ with discrete topology are amenable hence so is $G$ with discrete topology.)

Proof. If $\operatorname{TLIM}(G)=\operatorname{LIM}(G)$ then $\operatorname{TLIM}\left(G_{0}\right)=\operatorname{LIM}\left(G_{0}\right)$ since $T v \in \operatorname{TLIM}(G)=\operatorname{LIM}(G)$ for all $v \in \operatorname{LIM}\left(G_{0}\right)$. Thus $v \in \operatorname{TLIM}\left(G_{0}\right)$ by the previous proposition. We use now Theorem 1 and get that $G_{0}$ is discrete. Thus if $x \in G_{0},\{x\}$ is open in $G_{0}$ hence in $G$. Hence $G$ is discrete.

The following is an interpretation of our and some known related results from the point of view of harmonic analysis on locally compact groups.

Let $H\left[H_{c}\right]$ denote the linear span of $\left\{f-l_{x} f ; f \in L^{\infty}(G), x \in G\right\}$ [\{f- $\left.\left.\phi * f ; f \in L^{\infty}(G), \phi \in P(G)\right\}\right]$. If $A \subset L^{\infty}(G)$ denote by $\bar{A}\left[\bar{A}^{*}\right]$ its norm [ $\left.w^{*}\right]$ closure.

We need the following known remark whose proof uses a trick due to I. Namioka [9].

Remark. Let $\Psi, \Psi_{1}, \Psi_{2} \in L^{\infty}(G)^{*}, \phi \in P(G)$ and define $\left(L_{\phi} \Psi\right) f=$ $\Psi(\phi * f)$ for $f \in L^{\infty}(G)$. Let $\Psi_{1} \vee \Psi_{2}=\max \left(\Psi_{1}, \Psi_{2}\right)$ in the lattice $L^{\infty}(G)^{*}$ and $\Psi^{+}=\Psi \vee O, \Psi^{-}=(-\Psi) \vee O$. If $\Psi \in L^{\infty}(G)^{*}$ satisfies $L_{\phi} \Psi=\Psi$ for all $\phi \in P(G)$, then so do $\Psi^{+}$and $\Psi^{-}$: If $\phi \in P(G), L_{\phi}(\Psi \vee O) \geqq\left(L_{\phi} \Psi \vee L_{\phi} O\right)=$ $\Psi \vee O=\Psi^{++}$. So $L_{\phi} \Psi^{+}-\Psi^{+} \geqq 0$. But $\left(L_{\phi} \Psi^{+}-\Psi^{+}\right)(1)=0$. Thus $L_{\phi} \Psi^{+}=\Psi^{+}$. (Same true, if $L_{\phi}$ is replaced by $l_{a}^{*}$ for all $a \in G$.)

Proposition 5. (a) Let $G$ be compact and infinite. Then

$$
\bar{H} \subset \bar{H}_{c}=\bar{H}_{c}^{*}=\bar{H}^{*}=\left\{f \in L^{\infty}(G) ; \lambda f=0\right\} .
$$

If $G$ is abelian (or even amenable as a discrete group) then $\bar{H} \neq \bar{H}_{c}$. 
(b) Let $G$ be a noncompact locally compact group. Then $\bar{H} \subset \bar{H}_{c} \subset \bar{H}^{*}=$ $\bar{H}_{c}^{*}=L^{\infty}(G)$. Furthermore

(i) $\bar{H}_{c}=L^{\infty}(G)$ iff $\bar{H}=L^{\infty}(G)$ iff $G$ is not amenable (i.e. LIM=ø).

(ii) If $G$ is $\sigma$-compact amenable then $L^{\infty}(G) / \bar{H}_{c}$ is a nonseparable Banach space.

(iii) If $G$ is a $\sigma$-compact and amenable as discrete or amenable and containing such an open normal subgroup (in particular if $G$ is locally compact abelian), then $\bar{H}=\bar{H}_{c}$ iff $G$ is discrete.

Proof. (a) $\bar{H} \subset \bar{H}_{c}$ is due to the fact that TLIM $\subset$ LIM [6, p. 25], the remark above and the Hahn-Banach theorem (this part with $G$ not necessarily compact). Thus $\bar{H}^{*} \subset \bar{H}_{c}^{*}$. If the inclusion were proper then there would exist some $\phi \in L_{1}(G)$ such that $\phi(H)=0$ but $\phi(g) \neq 0$ for some $g \in H_{c}$. But then $\phi$ is left invariant and in $L_{1}(G)$ hence $\phi=c \lambda$ for some scalar $c \neq 0$. Hence $\phi\left(H_{c}\right)=\lambda\left(H_{c}\right)=0$ which cannot be. So $\bar{H} \subset \bar{H}_{c} \subset \bar{H}_{c}^{*}=$ $\bar{H}_{c}^{*} \subset\left\{f \in L^{\infty}(G) ; \lambda f=0\right\}$.

That $\bar{H}_{c}=\left\{f \in L^{\infty}(G) ; \lambda f=0\right\}$ is a consequence of Theorem 7.3, p. 360 of J. C. S. Wong [10] or can directly be proven. The rest of (a) is implied by the main theorem of this paper.

(b) If $\bar{H}^{*} \neq L^{\infty}(G)$ there would exist $0 \neq \phi \in L_{1}(G)$ such that $\phi(H)=0$. But then $\phi$ is left invariant hence so are $\phi^{+}, \phi^{-}$and $\phi^{+} \neq 0$ or $\phi^{-} \neq 0$. Assuming that $\phi^{+} \neq 0, \mu(A)=\int_{A} \phi^{+} d \lambda$ is a measure on the Borel sets of $G$ satisfying all the conditions in Hewitt-Ross [7, p. 194]. Hence $\mu=c \lambda$ for some $c>0$ (since $\mu \neq 0)$.

Since $\mu(G)<\infty, \lambda(G)<\infty$ so $G$ is compact. That (b)(i) holds is known and readily shown. (b)(ii) is shown as follows: If $L^{\infty}(G) / \bar{H}_{c}$ would be separable there would exist a sequence $\left\{f_{n}\right\} \subset L^{\infty}(G)$ such that (if $B$ is the linear span of $\left.\left\{f_{n}\right\}\right) \bar{H}_{c}+B$ is norm dense in $L^{\infty}(G)$ (see $[4$, p. 63]). But $\bar{H}_{c}=\left\{f \in L^{\infty}(G) ; \Psi(f)=0\right.$ for all $\Psi \in$ TLIM $\}$ Wong [10, p. 360]. Fix now some $\Psi_{0} \in$ TLIM and let $\Psi_{0} f_{n}=\alpha_{n}$. Then $\left\{\Psi_{0}\right\}=\{\Psi \in$ TLIM; $\left.\Psi f_{n}=\alpha_{n} n \geqq 1\right\}$ since any $\Psi$ which belongs to the right side will coincide with $\Psi_{0}$ on $\bar{H}_{c}+B$ hence on $L^{\infty}(G)$. We apply now [4, Theorem 5, p. 53] with $K=P(G)$ hence $A=\left\{\Psi^{\circ} \in \mathrm{TLIM} ; \Psi^{\prime}\left(f_{n}-\alpha_{n}\right)=0\right\}=\left\{\Psi_{0}\right\}$ is norm separable. Thus $G$ is compact. (b) (iii) is just our main theorem and the fact that $\bar{H}=\bar{H}_{c}$ iff LIM=TLIM (by our remark above and the HahnBanach theorem).

Main CONJeCture. Let $G$ be any amenable locally compact group. If $G$ is noncompact then $L^{\infty}(G) / \bar{H}_{c}$ is nonseparable. If $G$ is nondiscrete then $\bar{H}_{c} / \bar{H}$ is nonseparable.

Addition. In the meantime W. Rudin sent us a preprint of a paper of his, in which he proves Theorem 2 without the assumption that $\left({ }^{*}\right)$ " $G$ contains an open $\sigma$-compact normal subgroup", but with the assumption 
that $G$ is amenable as discrete. His proof is different from ours and uses harmonic analysis type arguments. After reading his manuscript we found the following easy argument which removes the restriction $\left({ }^{*}\right)$.

Proposition. Let $G_{0}$ be an open noncompact subgroup of $G$, and

$$
G=\bigcup_{\alpha \in I} x_{\alpha} G_{0}, \quad x_{\alpha} G_{0} \cap x_{\beta} G_{0}=\varnothing \quad \text { if } \alpha \neq \beta .
$$

If $A_{0} \subset G_{0}$ is such that $\lambda\left(A_{0}\right)<\infty(\lambda$-the Haar measure on $G)$ then for all $\phi \in \mathrm{TRIM}, \phi\left(\bigcup_{\alpha \in I} x_{\alpha} A_{0}\right)=0$.

Proof. Let $B_{n} \subset G_{0}$ be compact with $\lambda\left(B_{n}\right)=a_{n} \uparrow \infty$ and let $f_{n}=a_{n}^{-1} 1_{B_{n}}$, $A=\bigcup_{\alpha} x_{\alpha} A_{0}$. Then

$$
\begin{aligned}
1_{A} * \tilde{f_{n}}(x) & =a_{n}^{-1} \int 1_{A}(y) \tilde{1}_{B_{n}}^{\tilde{y}}\left(y^{-1} x\right) d y \\
& =a_{n}^{-1} \lambda\left(x B_{n} \cap A\right) \leqq a_{n}^{-1} \lambda\left(x G_{0} \cap A\right) \\
& =a_{n}^{-1} \lambda\left(x_{x} G_{0} \cap A\right)=a_{n}^{-1} \lambda\left(x_{x} A_{0}\right)=a_{n}^{-1} \lambda\left(A_{0}\right),
\end{aligned}
$$

for some (hence all) $\alpha \in I$.

If $\phi \in$ TRIM then $\phi(A)=\phi\left(1_{A} * f_{n}^{-}\right) \leqq a_{n}^{-1} \lambda\left(A_{0}\right) \rightarrow 0$.

RemarK. If $\Psi \in$ TLIM, then $\psi\left(\bigcup_{\alpha} A_{0}^{-1} x_{\alpha}^{-1}\right)=0$. (See [4, pp. 49-50].) To remove restriction $\left({ }^{*}\right)$ on $G$, let $G_{0}$ be any $\sigma$-compact, noncompact, open subgroup of $G$, if $G$ is noncompact, and $G=G_{0}$, if $G$ is compact. Let $A_{0} \subset G_{0}$ be open dense with $\lambda\left(A_{0}\right) \leqq \frac{1}{2}$ and $A=\bigcup_{\alpha} A_{0}^{-1} x_{\alpha}^{-1}$ ( $x_{\alpha}$ as above), $A=A_{0}$ if $G$ is compact. Let $B=G \sim A$. Then $\psi(B)=1$ for all $\psi \in$ TLIM, if $G$ is not compact, $\lambda(B) \geqq \frac{1}{2}$ if $G$ is compact. $B$ is closed nowhere dense. Continue now as in the proof of Theorem 1 .

\section{REFERENCES}

1. M. M. Day, Amenable semigroups, Illinois J. Math 1 (1957), 509-544. MR 19, 1067.

2. E. Følner, On groups with full Banach mean values, Math. Scand. 3 (1955), 243-254. MR 18, 51.

3. - Note on groups with and without full Banach mean value, Math. Scand. 5 (1957), 5-11. MR 20 \#1237.

4. E. Granirer, Exposed points of convex sets and weak sequential convergence, Mem. Amer. Math. Soc. No. 123 (1972).

5. - On finite equivalent invariant measures for semigroups of transformations, Duke Math. J. 38 (1971), 395-408. MR 44 \#404.

6. F. P. Greenleaf, Invariant means on topological groups and their applications, Van Nostrand Math. Studies, no. 16, Van Nostrand Reinhold, New York, 1969. MR 40 \#4776. 
7. E. Hewitt and K. Ross, Abstract harmonic analysis, Vol. I: Structure of topological groups. Integration theory, group representations, Die Grundlenren der math. Wissenschaften, Band 115, Academic Press, New York, Springer-Verlag, Berlin, 1963. MR 28 \#158.

8. I. Namioka, Folner's conditions for amenable semigroups, Math Scand. 15 (1964), 18-28. MR 31 \#5062.

9. - On certain actions of semigroups on L-spaces, Studia Math. 29 (1967), 63-77. MR 36 \#6910.

10. J. C. S. Wong, Topologically stationary locally compact groups and amenability, Trans. Amer. Math. Soc. 144 (1969), 351-363. MR 40 \#2781.

Department of Mathematics, University of British Columbia, Vancouver, British Columbia, Canada 